DOI: https://doi.org/10.11144/Javeriana.umed60-3.cbsu

\title{
Conceptos bioéticos para tener en cuenta en el servicio de urgencias
}

\section{Some Bioethical Concepts to Consider in the Emergency Room}

Recepción: 12/02/2019 | Aceptación: 28/03/2019

\author{
Atilio Moreno Carrillo \\ Hospital Universitario San Ignacio, Colombia \\ María Paola Aguilar Rodas \\ Pontificia Universidad Javeriana, Colombia
}

\footnotetext{
a Correspondencia: amoreno@husi.org.co;

atilio.moreno@javeriana.edu.co
}

Cómo citar: Moreno Carrillo A, Aguilar Rodas MP. Conceptos bioéticos para tener en cuenta en el servicio de urgencias. Univ Med. 2019;60(3). https:// doi.org/10.11144/Javeriana.umed60-3.cbsu

\section{RESUMEN}

En el campo de la medicina, y más aún en el servicio de urgencias, el médico se encuentra constantemente expuesto a dilemas éticos, los cuales, sin una base conceptual firme, son difíciles de solucionar. Aunque existen normativas y principios éticos establecidos, como la beneficencia, no maleficencia, autonomía y justicia, además de ciertos códigos, por ejemplo, el Código de Ética del Colegio Americano de Emergenciólogos, no existe una estandarización en esta práctica, porque las situaciones pueden ser tan variadas como los pacientes. El presente artículo pretende analizar las circunstancias más frecuentes y dar algunas bases bioéticas para seguir en estos casos.

Palabras clave

bioética; urgencias; decisiones.

\begin{abstract}
In the field of medicine and even more in the emergency room physicians are constantly exposed to ethycal dilemas wich without solid concepts are hard to solve, eventhough there are some principles like: beneficence, non-maleficence, autonomy and justice, and also codes like the American College of Emergency Physicians Code of Ethics for emergency physicians, there is not an standard in this practice because such situations are as variable as patients. In this article we analyze the most common circumstances and we give some basis to follow on this cases.
\end{abstract}

Keywords

bioetical; emergency; decisions.

\section{Introducción}

La relación médico-paciente está regida tanto por consideraciones legales, éticas y morales como por normatividad; además, todo cuanto se haga en los servicios de salud se debe realizar a favor de los enfermos. Los médicos están obligados a hacer parte del actuar diario con sus pacientes los principios éticos de autonomía, beneficencia, justicia y no maleficencia. Como deber profesional y respetando los derechos de los pacientes, toda decisión sobre 
estos exige previo un consentimiento informado, tomado en un paciente que ha recibido toda la información pertinente y que tenga la capacidad de consentir.

Son muchos los desafíos a los que se enfrentan los médicos de emergencias, entre ellos los morales listados en la tabla 1 (1). Todos los dilemas citados en la tabla constituyen situaciones del diario vivir en el servicio de urgencias, lo que refleja la complejidad de la labor en este servicio, tanto desde el punto de vista del paciente como por el entorno donde se trabaja y la sociedad.

\section{Tabla 1}

Desafíos morales de los médicos de emergencia

1. Pacientes con enfermedades agudas o lesiones que
requieren atención inmediata.
2. Pacientes incapaces de participar en las decisiones
relativas a su salud, debido a los cambios agudos en
su estado mental.
3. No contacto previo con los pacientes.
4. Pacientes en crisis en ocasiones sin aceptar su
ingreso a urgencias.
5. Entorno de trabajo institucional con variedad de
profesiones.
6. Responsabilidad social como proveedores de
atención de salud para pacientes que no tienen fácil
acceso a la atención sanitaria.
7. Deber social de actuar fuera de su entorno normal
de atención de la salud cuando hay situaciones en
donde su intervención puede salvar vidas.
8. Ser un recurso para la comunidad en la atención
extrahospitalaria para manejo de desastres,
toxicologia, reanimación cardiopulmonar, salud
pública, entre otras.

Fuente: adaptada de Código de Ética para Médicos de Emergencia del Colegio

Americano de Médicos de Emergencia, 2017.

La humanización en la salud es un concepto fundamental, porque el paciente que ingresa a urgencias se encuentra, de por sí, en una condición de vulnerabilidad; incluso, en algunos casos, en situación de inferioridad respecto al personal médico, por diversas causas que pueden relacionarse desde el nivel intelectual hasta su condición de salud. El advenimiento de grandes corporaciones $\mathrm{o}$, aun, la mercantilización de la salud han contribuido a que esta se vea deshumanizada (2). En el servicio de urgencias, el profesional de la salud se encuentra ante un reto todavía mayor frente a este concepto, debido a todas las limitantes ya conocidas, como lo son tiempo, espacio físico, disponibilidad de servicios, etc.

Un gran desafío para los médicos de urgencias lo representa el saber abordar las necesidades de los pacientes con enfermedades terminales, respetando los deseos de limitar las intervenciones (3).

Otro aspecto destacado es que aun cuando existen principios y códigos claros, estos no siempre son aplicables, debido a la realidad de los servicios de salud en Latinoamérica y, sobre todo, urgencias. La demanda de este servicio en hospitales públicos y privados es muy alta, lo cual puede tener relación con las características del sistema de salud, que hacen que se deban tratar en este escenario no solo los casos graves que amenazan la vida, sino también enfermedades que regularmente deberían ser atendidas en consulta externa (4). La congestión y las condiciones en las que se debe atender a los pacientes en urgencias no siempre son las óptimas, por lo que es complicado aplicar los principios mencionados.

El gran dilema en los servicios de emergencias se puede resumir en responder a las preguntas: icómo se evalúa la capacidad de toma de decisiones en los enfermos críticos en un servicio de emergencia? ¿Cómo asegurar que el paciente entienda la información para la toma de decisiones, la retenga, la piense, decida y pueda comunicar su decisión ante una situación crítica? En el presente artículo se pretende poner en consideración los principios y dilemas éticos, y revisar la normatividad existente en el momento $(5,6)$.

\section{Principios éticos en el ámbito de las unidades de emergencias}

Según el Código de Ética para Médicos de Emergencia del Colegio Americano de Médicos de Emergencia (7), los médicos de emergencias deben actuar en consonancia con los principios éticos explicados a continuación: 
Beneficencia. Teniendo en cuenta que los médicos de las unidades de emergencias tienen como objetivo prevenir la muerte de un paciente o minimizar los daños de su patología, se entiende que actúan bajo el principio de la beneficencia. Adicionalmente, al proteger la privacidad del paciente, manteniendo la confidencialidad de la información que dé y la obtenida por medio de la historia clínica, también están actuando según este principio.

No maleficencia. Los médicos de urgencias no deben iniciar tratamientos que puedan causar más daño que beneficio y deben procurar maximizar los beneficios del tratamiento y minimizar el riesgo de daño.

Autonomía. Los pacientes adultos con capacidad para tomar decisiones tienen derecho a aceptar o rechazar la atención médica recomendada, y los médicos tienen el deber de respetarlas, no sin antes haber dado información precisa sobre sus condiciones médicas y las opciones de diagnóstico y tratamiento. Este derecho se materializa en el consentimiento informado. Si el paciente carece de capacidad para tomar decisiones, los médicos de emergencia también deben respetar las decisiones médicamente razonables tomadas por el responsable de la toma de decisiones del paciente.

Los médicos de emergencia deben ser expertos en determinar la capacidad de toma de decisiones y en identificar a los responsables apropiados de la toma de decisiones. En el ámbito de las urgencias hay excepciones limitadas para actuar sobre el paciente sin el consentimiento informado, como necesidad de una intervención inmediata para prevenir la muerte o daño grave al paciente, cuando el paciente carece de capacidad para tomar decisiones, cuando no hay nadie legalmente autorizado para consentir en nombre del paciente y cuando el tratamiento es necesario para proteger la salud pública.

Los pacientes pueden, si lo desean, renunciar a su derecho al consentimiento informado o delegar en otros la autoridad de toma de decisiones para su cuidado. Otro aspecto importante es la atención de pacientes terminales. Los médicos de urgencia deben estar dispuestos a respetar el deseo de un paciente en estado terminal de renunciar al tratamiento que prolonga la vida, expresado en un testamento en vida o por un representante apropiado.

Justicia. Los médicos de emergencia tienen un deber de justicia para proporcionar atención a los pacientes independientemente de raza, color, credo, género, nacionalidad, entre otros: dar a cada persona lo que es debido. En Estados Unidos, la política pública ha establecido un derecho limitado de los pacientes a recibir tratamiento de evaluación y estabilización para las condiciones médicas de emergencia en los departamentos de emergencia de los hospitales. En Colombia, existe un nuevo plan de beneficios en salud, para la atención en salud de todos los afiliados al Sistema General de Seguridad Social en Salud a que tienen derecho (8). Adicionalmente, según el Código de Ética para Médicos de Emergencia, los médicos de emergencia tienen el deber en la justicia de actuar como administradores responsables de los recursos de atención de salud que se les confían (6).

\section{Toma de decisiones compartidas en unidades de emergencia}

La toma de decisiones compartidas (TDC), entendida como "el proceso colaborativo que permite a los pacientes, sus sustitutos y los médicos tomar decisiones de salud juntos, teniendo en cuenta la mejor evidencia científica disponible, así como los valores, metas y preferencias del paciente" (9), hace parte del proceso en el que se desarrolla la relación médico-paciente, en consonancia con los principios éticos en los que se basa tal relación. Se han sugerido características clave para la TDC: que al menos dos participantes estén involucrados (médico y paciente), que ambas partes compartan información, que ambas partes se involucren para construir el consenso sobre el tratamiento preferido y que haya acuerdo acerca del tratamiento que se vaya a implementar (10).

Para implementar el concepto de TDC en urgencias es importante tener en cuenta uno 
de los derechos fundamentales del paciente: la comunicación plena y clara con el médico que le permita tomar la mejor decisión. Para aplicar este concepto, la base es que se le dé al paciente toda la información de forma clara, cerciorarse de que haya comprendido y guiarlo en tomar la decisión acorde con sus intereses, creencias personales, etc. $(7,8)$.

También se ha estudiado el tema de la TDC en sectores críticos en la atención sanitaria, por ejemplo, las unidades de cuidado intensivo. En este contexto, se encuentran recomendaciones entre las cuales están usar un proceso de TDC que incluya el intercambio de información, deliberación y toma de decisiones de tratamiento.

En la TDC hay amplia gama de enfoques que incluyen los modelos dirigidos por los pacientes o sustitutos o dirigidos por los clínicos, que se debe adaptar teniendo en cuenta las preferencias del paciente o sus sustitutos. También es necesario entrenar a los médicos en habilidades de comunicación y amplia investigación para evaluar las estrategias en la TDC (11).

En otras situaciones críticas, como la atención en urgencias, la premura en la toma de decisiones para salvar la vida del paciente representa un gran desafío para no dejar de lado los principios éticos de autonomía, beneficencia, no maleficencia y justicia durante todo el proceso de atención desde la toma de pruebas diagnósticas, pasando por las intervenciones terapéuticas hasta el seguimiento intrahospitalario. En la tabla 2 se muestra un modelo propuesto para orientar las decisiones en cuanto a la toma de pruebas diagnósticas (12).

\section{Tabla 2}

Pruebas diagnósticas en el departamento de emergencias: un modelo para la toma de decisiones

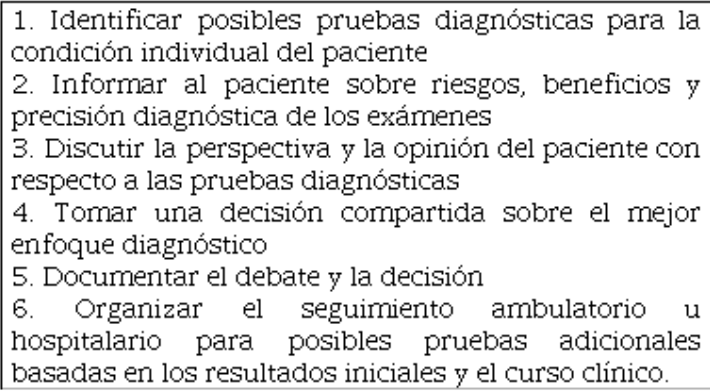
condición individual del paciente

2. Informar al paciente sobre riesgos, beneficios y precisión diagnóstica de los exámenes

3. Discutir la perspectiva y la opinión del paciente con respecto a las pruebas diagnósticas

4. Tomar una decisión compartida sobre el mejor enfoque diagnóstico

5. Documentar el debate y la decisión

6. Organizar el seguimiento ambulatorio u hospitalario para posibles pruebas adicionales basadas en los resultados iniciales y el curso clínico.

Fuente: adaptada de Kraus CK, Marco

CA. Shared decision making in the ED: ethical considerations, 2016.

Ahora bien, respecto a las intervenciones terapéuticas, lo ideal sería que se estableciera también la TDC teniendo en cuenta la autonomía y la beneficencia del paciente; sin embargo, en situaciones críticas el juicio clínico tendrá prioridad, por ejemplo, cuando el estado mental del paciente está alterado y no es posible entablar un diálogo. Para estos desafíos que representan, el paciente sin capacidad de decidir y sin un representante (legal, familiar, entre otros) se recomienda, en lo posible, conocer sus preferencias personales o directivas anticipadas. También se ha propuesto que, de no conocerse sus preferencias, se decida con base en las preferencias conocidas de pacientes que compartan características con el paciente como edad, sexo y antecedentes clínicos, que constituiría un indicador de tratamiento basado en la población y que podría predecir las preferencias de los pacientes con la misma precisión que los sustitutivos designados por los pacientes y los familiares (13).

Se recomienda realizar el máximo intento razonable para hacer partícipe al paciente y sus subrogados en la toma de decisiones como responsabilidad ética frente a las complejas situaciones en las unidades de emergencias (14).

Hay factores especiales que influyen en la TDC y en la relación médico-paciente que pueden generar barreras en una unidad de urgencias. Del paciente, como gravedad de la 
enfermedad, capacidad y voluntad para la toma de decisiones; nivel educativo y socioeconómico. De los familiares, como patrones de práctica, voluntad de participar en el proceso de toma de decisiones y percepciones médicolegales. Del propio sistema de salud y hasta limitaciones en la evidencia científica disponible para tratar al paciente. No obstante, pese a que los modelos de TDC en salud se han conceptualizado para situaciones ambulatorias, se plantea que el médico de emergencias empiece tomando decisiones frente a un modelo paternalista, es decir, sea él quien vele por el bienestar del paciente: tomando inicialmente pruebas diagnósticas básicas, dando tratamiento sintomático $y$, posteriormente, una vez que el paciente o sus acompañantes pueden participar, seguir con los modelos compartidos e informados, haciendo que el paciente y sus acompañantes tomen las riendas en las decisiones (15). Aunque son pocos los estudios que han explorado la TDC en las unidades de emergencias, no hay evidencia disponible que sugiera que se no pueda tener éxito (16).

\section{Códigos de ética y legislación}

Los códigos de ética internacionales, como la Declaración de Derechos del Paciente de la Asociación Americana de Hospitales y el Convenio Europeo de Bioética, concuerdan en que, frente a una situación de urgencia, en la que el paciente no esté en condiciones de dar su consentimiento informado, se puede proceder a cualquier intervención a favor de la vida del paciente (17).

En Colombia, la legislación al respecto es similar, aunque superficial en cuanto a las directrices dadas en situaciones de urgencias. La Ley de Ética Médica (Ley 23 de 1981), que dicta normas en materia de ética médica para el país, establece en su artículo 14 que: "el médico no intervendrá quirúrgicamente a menores de edad, a personas en estado de inconsciencia o mentalmente incapaces, sin la previa autorización de sus padres, tutores o allegados, a menos que la urgencia del caso exija una intervención inmediata".

En el artículo 26, "El médico no prestará sus servicios profesionales a personas de su familia o que de él dependan en casos de enfermedad grave o toxicomanía, salvo en aquellas de urgencia o cuando en la localidad no existiere otro médico". Y en cuanto a la relación médicopaciente, en el artículo 5: "La relación médicopaciente se cumple en los siguientes casos; 1 . Por decisión voluntaria y espontánea de ambas partes. 2. Por acción unilateral del médico, en caso de emergencia. 3. Por solicitud de terceras personas. 4. Por haber adquirido el compromiso de atender a personas que están a cargo de una entidad privada o pública" (18).

Posteriormente, se reglamentó esta ley con el Decreto 3380 de 1981, de la cual se pueden extraer los siguientes apartados relacionados con el tema de la atención en urgencias: Artículo $3^{\circ}$ : "Para señalar la responsabilidad médica frente a los casos de emergencia o urgencia, entiéndase por esta todo tipo de afección que ponga en peligro la vida o integridad de la persona y que requiera atención inmediata de acuerdo con el dictamen médico".

Artículo 11: "El médico quedará exonerado de hacer la advertencia del riesgo previsto en los siguientes casos: a) Cuando el estado mental del paciente y la ausencia de parientes allegados se lo impidan. b) Cuando existe urgencia o emergencia para llevar a cabo el tratamiento o procedimiento médico" (19).

En 1991, con la Resolución 13.437 de 1991, con la que se constituyen los comités de ética hospitalaria y se adopta el Decálogo de los Derechos de los Pacientes, se encuentra respecto a la atención en urgencias, en el artículo 1:

Adoptar como postulados básicos para propender por la humanización en la atención a los pacientes y garantizar el mejoramiento de la calidad en la prestación del servicio público de salud en las Instituciones Hospitalarias Públicas y Privadas, los Derechos de los pacientes que se establecen a continuación: numeral $6 \mathrm{Su}$ derecho a revisar y recibir explicaciones acerca de los costos por los servicios obtenidos, tanto por parte de los profesionales de la salud como 
por las instituciones sanitarias. Al igual que el derecho a que en casos de emergencia, los servicios que reciba no estén condicionados al pago anticipado de honorarios. (19)

En lo que respecta a directrices específicas para las situaciones de emergencia, existe un Código de Ética para Médicos de Emergencia del Colegio Americano de Médicos de Emergencia, en el que se expresan las responsabilidades fundamentales de los médicos de emergencia resumidas en la tabla 3 .

\section{Tabla 3}

Principios de ética para los médicos de emergencia 1. El bienestar del paciente es la principal responsabilidad profesional
2. Responder con prontitud y habilidad, sin prejuicio o parcialidad, a la necesidad de la
atención médica de emergencia
3. Respetar los derechos y esforzarse por proteger los mejores intereses de sus
pacientes, en particular los más vulnerables y los que tienen una capacidad de toma de
decisiones deficiente
4. Comunicarse de manera veraz con los pacientes y obtener su consentimiento
informado para el tratamiento, a menos que la urgencia del paciente requiera una
respuesta inmediata u otra excepción establecida para obtener el consentimiento
informado
5. Respetar la privacidad del paciente y revelar información confidencial solo con el
consentimiento del paciente o cuando así lo requiera un deber primordial (proteger a
otros o aspectos legales)
6. Tratar de manera justa y honesta a sus colegas y tomar las medidas apropiadas para
proteger a los pacientes de los proveedores de atención médica incompetentes, o que
estén involucrados en fraude o engaño
7. Trabajar en cooperación con otros que cuidan y tratan a los pacientes de emergencia
8. Mantener conocimientos actualizados y las habilidades necesarias para proporcionar
atención de alta calidad a los pacientes
9. Actuar como administradores responsables de los recursos de atención de la salud
10. Apoyar los esfuerzos de la sociedad para mejorar la salud pública y la seguridad,
reducir los efectos de lesiones y enfermedades, y asegurar el acceso a la atención de
salud de emergencia y de otra indole para los pacientes

Fuente: adaptada de Código de Ética para Médicos de Emergencia del Colegio Americano de Médicos de Emergencia, 2017.

Estos principios orientan de forma más expedita el actuar de los médicos y pudieran servir de base para adaptarlos a nuestro medio, en vista de que las disposiciones éticas existentes en el momento son generales.

\section{Conclusiones}

El médico de urgencias se encuentra expuesto a dilemas éticos de forma constante que, por diversos motivos, como circunstancias relativas al pacientes, el entorno y las situaciones legales, constituyen un verdadero desafío.

Los diferentes códigos disponibles proponen que el actuar médico se encuentre dentro del marco de los principios éticos fundamentales que rigen su práctica; adicionalmente, siempre que se pueda, se utilice una estrategia de TDC, incluso, aunque las decisiones iniciales puedan obligarnos a obviar este paso, se propenda, una vez estabilizado el paciente, a otorgarle autonomía en las decisiones y la estructuración del consentimiento informado.

Otro punto para considerar es la relación con los familiares, allegados o quien quiera que sea el acompañante del paciente, ya que ellos son quienes en ocasiones nos aportan la información o incluso son quienes tomarán las decisiones por el paciente. Si bien en ocasiones es complejo hacerlos parte del proceso - principalmente por el limitado espacio en los servicios de urgencias -, es importante que sean parte de la TDC y aplicar en ellos los conceptos de humanización y los principios éticos básicos, tal cual se hace con el paciente.

Dentro de las normativas locales se evidencian vacíos en los cuales el médico puede encontrar dificultades para su actuar. Existen múltiples sentencias dadas por la Corte Constitucional, como la T-760 del 2008, la cual detalla los derechos de los pacientes, sobre los cuales se puede sentar una base para la aplicación de los principios éticos. Sería imposible realizar un manual donde se detallen todas las situaciones y escenarios posibles, por lo que se recomienda actuar siempre en beneficio del paciente.

\section{Referencias}

1. Díaz A. La humanización en salud conceptos, críticas y perspectivas. Bogotá: Editorial Pontificia Universidad Javeriana; 2017.

2. Zieske M, Abbott J. Ethics seminar: the hospice patient in the ED: an ethical approach to understanding barriers and improving care. Acad Emerg Med. 2011 Nov;18(11):1201-7.

3. Ardila S, Velazco N, Jiménez A, Amaya C. Revisión de procesos para la asignación de camas a pacientes provenientes de urgencias de un hospital privado de Bogotá. Bogotá: Universidad de los Andes; 2008. 
4. Pelluchon C. El ejercicio de la medicina: valores de los pacientes, normas de los profesionales, conflictos y deliberación pública. Rev Colomb Bioet. 2011;6(2):139-59. http://dx.doi. org/10.18270/rcb.v6i2.833

5. Kottow M. Participación informada en clínica e investigación biomédica: las múltiples facetas de la decisión y el consentimiento informados. Bogotá: Unesco-Unibiblos; 2007.

6. American College of Emergency Physicians. Code of ethics for emergency physicians [internet]; 2009. Disponible en: http://www.acep.org/pr actres.aspx? $\mathrm{id}=29144$

7. Resolución 6408 de 2016, por la cual se modifica el Plan de Beneficios en Salud con cargo a la Unidad de Pago por Capitación (UPC) [internet]. Disponible en: https://www.minsalud.gov.co/Nor matividad_Nuevo/Resoluci\%C3\%B3n\% 206408\% 20 de\%202016.pdf.

8. Kon AA, Davidson JE, Morrison W, Danis M, White DB. Shared decision making in ICUs: an American College of Critical Care Medicine and American Thoracic Society Policy Statement. Crit Care Med 2016;44(1):188-201.

9. Charles C, Gafni A, Whelan T. Shared decision-making in the medical encounter: what does it mean? (Or it takes at least two to tango). Soc Sci Med. 1997 Mar;44(5):681-92.

10. Kon AA, Davidson JE, Morrison W, Danis M, White DB. Shared decision making in intensive care units: an American College of Critical Care Medicine and American Thoracic Society Policy Statement. Crit Care Med. 2016 Jan;44(1):188-201.

11. Kraus CK, Marco CA. Shared decision making in the ED: ethical considerations. Am J Emerg Med. 2016 Aug;34(8):1668-72.
12. Varma S, Wendler D. Medical decision making for patients without surrogates. Arch Intern Med 2007;167(16):1711-5.

13. Kraus CK, Marco CA. Shared decision making in the ED: ethical considerations. Am J Emerg Med. 2016 Aug;34(8):1668-72.

14. Hess EP, Grudzen CR, Thomson R, Raja AS, Carpenter CR. Shared decision-making in the emergency department: respecting patient autonomy when seconds count. Acad Emerg Med 2015;22:856-64.

15. Flynn D, Knoedler MA, Hess EP, et al. Engaging patients in health care decisions in the emergency department through shared decision-making: a systematic review. Acad Emerg Med. 2012;19:959-67.

16. Escobar Triana J. Convenios y declaraciones de ética médica, enfermería y bioética. Bogotá: Ediciones El Bosque; 1998.

17. Ley 23/1981 del 18 de febrero, por la cual se dictan normas en materia de ética médica [internet]. Diario Oficial 35.711, de 27 de febrero de 1981. Disponible en: https://www.icbf.gov.co/cargues/av ance/docs/ley_0023_1981.htm.

18. Decreto 3380/1981 del 30 de noviembre, por el cual se reglamenta la Ley 23 de 1981 [internet]. Diario Oficial 35914, del 30 de diciembre de 1981. Disponible en: https://www.mineducacion.gov.co/ 1621/articles-103328_archivo_pdf.pdf

19. Resolución 13437/1991, por la cual se constituyen los comités de ética hospitalaria y se adoptan el Decálogo de los Derechos de los Pacientes [internet]. Ministerio de Salud. Disponible en: https://www.minsalud.gov.co/Nor matividad_Nuevo/RESOLUCI\%C3\%93 N\%2013437\%20DE\%201991.pdf 